\title{
Impact of market-coupling on electricity price modeling in fundamental unit-commitment approaches
}

\author{
Moritz Nobis (), Lothar Wyrwoll, Albert Moser \\ Institute for High Voltage Equipment and Grids, \\ Digitalization and Energy Economics (IAEW) \\ RWTH Aachen University \\ Aachen, Germany \\ moritz.nobis@rwth-aachen.de
}

\author{
Stephan Raths \\ Amprion $\mathrm{GmbH}$ \\ Puhlheim, Germany \\ stephan.raths@amprion.net
}

\begin{abstract}
Fundamental unit commitment approaches are of central importance in energy system modeling for the generation of detailed power plant schedules. However, existing approaches, which reduce complexity in a multi-stage process, often fail to generate realistic electricity prices. A new type of singlestage approach considers market-coupling implicitly so that, in addition to detailed power plant schedules, electricity prices reflecting real prices very well can be generated. In this paper, we show in a back-test for 2014 that an endogenously modeled market-coupling is the driving factor for the quality of resulting electricity prices. Conversely, it can be concluded that conventional multi-stage approaches show a significant distortion of modeled electricity prices due to missing price signals from neighboring market zones. Against the background of expanding trading capacities between market zones within the European power system, this issue becomes increasingly relevant when fundamentally modeling energy prices.
\end{abstract}

Index Terms-electricity price modeling, Lagrangian relaxation, market-coupling, unit commitment

\section{INTRODUCTION}

Fundamental unit commitment (UC) models are an integral part of energy system planning. They are used an endogenous model component in expansion planning, to assess the influence of an expansion decision on systemic constraints (load coverage constraint, $\mathrm{CO}_{2}$ targets, etc.) [1]. Unit commitment models are also used in the evaluation of network expansion options, e.g. by the influence of adding a network element on resulting redispatch quantities, as in the network planning process of TSO. Another area of application is the subsequent financial evaluation of assets (power plants, electrolyzers, etc.) in a future energy system. Especially in the latter, electricity prices derived from modeling play a central role.

Unit Commitment approaches are characterized by their high level of detail and, due to their mixed-integer nature, a high degree of computational complexity. To model the behavior of power plants within the European interconnected power system it is necessary to model all (larger) power plants in all interconnected market areas. This leads to a large optimization problem with complex constraints and many integer decision variables, which as a closed problem cannot be solved even on large compute clusters. For this reason, such problems are often initially solved in a highly simplified way for Europe as a whole and then, decoupled, re-optimized in the individual market areas. For this purpose, the optimization problem is first reformulated into a linear optimization problem, so that only rudimentary power plant properties can be considered [2] [3] [4] [5]. In some cases, power plants outside the focus market area are aggregated into groups and thus also mapped in a highly simplified way [6] [7]. The determined exchange schedules are fixed and then the market areas are reoptimized using unit commitment approaches. Market areas are usually linked via NTC models, which allow for a direct cross-border-trade between two market zones while neglecting the electrical implications of the changing netpositions in those markets. Considering flow-based approaches in such large-scale optimization models is less common due to rising complexity and high sensitivity on associated flow-based parameters, and yet only considered in a few approaches [5]. If a Lagrangian approach is used for the second stage [2] [3] [4], an electricity price signal can be derived from the Lagrangian multiplier. This can have a high relevance within energy system modeling, as it is decisive both on the supply side for the profitability of assets and on the consumer side for the assessment of the burden of political measures (e.g. coal exit).

The solution of the optimization problem via multi-stage approaches has the disadvantage that the detailed power plant characteristics cannot influence the commitment decision across market areas. For example, start-up costs cannot have influence on international electricity trading. This distorts the resulting international trading volumes. Furthermore, bidding tuples of power plants in neighboring zones do not influence the merit order and thus the price formation in the zone under consideration. The aforementioned price distortion has already been the subject of scientific investigation in the past, but instead of questioning the model's architecture, only a post adjustment of prices was proposed [8]. In contrast, a new decomposition approach allows the optimization problem to be solved in a single-stage while taking market coupling 
into account so that the above-mentioned distortions do not occur [9].

We show in a back-test for the year 2014 that the integration of market coupling according to EUPHEMIA, the market coupling algorithm used in the European interconnected power system, into the unit commitment decision leads to electricity prices behaving like empirical electricity prices [10].

\section{Multi-Stage VS. Single-STAGE UC-APPROACHES}

The market simulations considered in this paper are aiming at generating individual power plant schedules in various considered market zones to cover the system's load. This can be realized through optimization problems that minimize the costs of the cross-system use of generation units. Assuming inelastic demand, this is analogous to maximizing market welfare. This problem can be formulated cohesively. Due to their computational complexity, the burden in those models are integer variables reflecting advanced technical power plant parameters. Moreover, it is not possible to infer market prices directly from the solution of a mixed-integer problem as they are usually derived from dual variables of the power balance constraints. There are different market simulation approaches to handle these challenges. In the following, a multi-stage approach which has been considered as state-of-the art for decades and the transition to a new single-stage approach will be discussed and explained.

The multi-stage-approach is based on a simplified model of all connected market areas $M$ and detailed consideration of individual market areas. The first stage aims at minimizing the total system costs which consists of the operational costs $c_{k}$ of all generation units $K$ while considering only simplified constraints. These are at least (2) keeping the power output of each unit between zero and the maximum output $\bar{P}$ and (3) restricting the gradient to range in between $P_{\downarrow}$ and $P_{\uparrow}$ :

$$
\begin{gathered}
\min _{p} \sum_{t \in T} \sum_{k \in K} c_{k} p_{k, t}, \quad p \in \mathbb{R}^{+} \\
0 \leq p_{k, t} \leq \bar{P}_{k}, \forall k \in K, t \in T \\
\text { s.t. } \quad P_{\downarrow} \leq p_{k, t}-p_{k, t-1} \leq P_{\uparrow}, \forall k \in K, t=2, \ldots, T
\end{gathered}
$$

This is subjected to a power-balance-constraint for each market area $m \in M$ in each time step $t \in T$ :

$$
D_{m, t}^{e l}=\sum_{k \in K_{m}} P_{k, t}+\sum_{j \in M} F_{n, m, t}-\sum_{j \in M} F_{m, n, t}
$$

The electrical load $D_{m}^{e l}$ is covered by feed-in of generation units $k$ and import flows $F_{n, m}$ minus export flows $F_{m, n}$. Those flows are limited by the respective net transfer capacity $\left(N T C_{n, m}\right)$ through 5 .

$$
0 \leq F_{m, n, t} \leq N T C_{m, n, t}, \forall m, n \in M, t \in T
$$

Due to its complexity, the optimization problem is formulated as a linear problem. Binary decision variables are therefore neglected.
In the second stage, all exchange flows $F$ are fixed by adding them to the load in the respective market zone. The operation of generation units in each market area is then reoptimized. This allows for adding binary variables to the resulting optimization problem and for applying unit commitment formulations, which include complex technical- and economical constraints [11]. This can be achieved through a closed formulation of the optimization problem or through applying Lagrangian relaxation. Here the load coverage in each market area is relaxed and the electricity price is used as Lagrangian multiplier $\lambda_{m}$. Since no electricity prices can be derived directly from such mixed-integer problems in a closed formulation, the Lagrangian decomposition enables the derivation of electricity prices through its approximated dual variable.

$$
\max _{\lambda_{m}}\left\{\min _{P} \sum_{t \in T} \sum_{k \in K_{m}} c_{k, t}+\sum_{t \in T} \lambda_{i}\left(D_{m, t}^{e l}-P_{k, t}\right)\right\}
$$

The Lagrangian multiplier $\lambda_{m}$ is iteratively adjusted until the constraints are fulfilled as best possible. However, deviations in the relaxed load covering constraint can remain due to linear cost coefficients of the objective function. Either the proposed $\lambda$ is greater or equal the marginal power plant costs than the resulting power plant bid is its nominal power, or the proposed $\lambda$ is below the marginal power plant costs resulting in a power plant shutdown (while neglecting some marginal aspects). These deviations are compensated in the third stage. The process of this market simulation approach is illustrated in Figure 1.

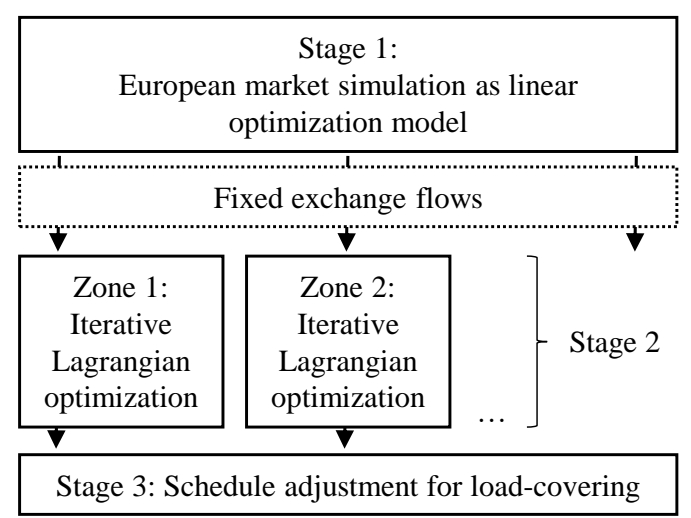

Fig. 1. Flow chart for the multi-stage-approach

Thus there is only limited influence of the UC-decision on exchange flows and no influence between Lagrangian multipliers across market zones.

The Lagrangian approach has been extended by including the EUPHEMIA market-coupling into (6). This approach, named European Lagrangian relaxation (EULR), allows for solving the problem in a single-stage [9]. Therefore, a predetermination of exchange flows is not necessary. In addition to relaxing the load constraint, potential imports and exports must 
also be taken into account in the adjustment of the Lagrangian multiplier. Thus, not only load coverage is a convergence criterion, but also by the use of exchange capacity. Prices converge if exchange capacities are not fully completed. This implies that price differences can only occur if exchange capacities are exhausted.

The optimization of generation schedules is analogous to formula (6) with the addition that $D_{m}^{e l}$, contains potential imports and exports. Based on the resulting schedules, the market-coupling is calculated analogously to the objective function in formula (1). Since the dispatch of generation units is optimized against the current value of the Lagrangian multiplier as market price, the respective price is also the cost coefficient.

Also in this approach, deviations in load coverage are possible despite maximum convergence and are remedied analogously. Figure 2 shows the process of this approach.

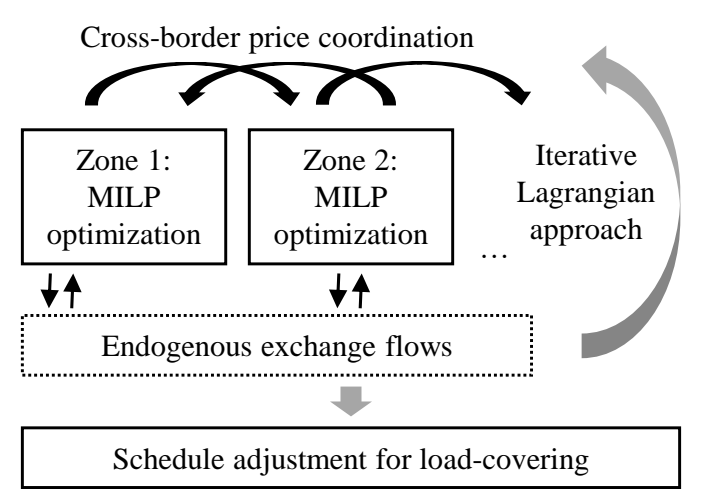

Fig. 2. Process of the approach in [9] for market simulation

Within the three-stage approach, a simplification by fixing the exchange flows is performed. The flows are derived from a linear problem and may not be entirely accurate for a detailed use of generation units, which may also distort prices. Furthermore, even with exact flows, a separate consideration of market zones with exogenous exchange cannot reflect correct market prices.

This is theoretically illustrated by the following example. We assume a merit order $S$ of two supply bids at a price of $\mathrm{P} 1$ and $\mathrm{P} 2$ and an inelastic demand D. The resulting market clearing price in the case without import and export is $\mathrm{P} 2$. If demand is reduced by the exogenous determination of imports (figure 3 left), the new market-clearing price is P1. However, the actual price at which imports are made can be in the range between P1 and P2 (figure 3 right). Bids from neighboring market zones must be included to model this effect. Thus, in the case of exogenously determined exchange flows, an underestimation of the price is possible.

In general, the problem occurs because the import is considered to be price neutral if its included exogenously. Since this does not reflect the actual market behavior, inaccurate prices can occur.
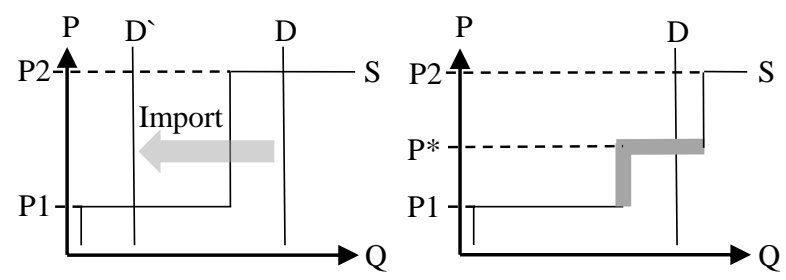

Fig. 3. Comparison of price determination for exogenous and endogenous exchange flows

\section{Simulated Electricity PRICES}

The multi-stage approach and the EULR are parameterized to meet 2014's energy market conditions and the model output is then compared with real market prices. The focus is on the German market area, whose empirical price distribution density is shown in figure 4.

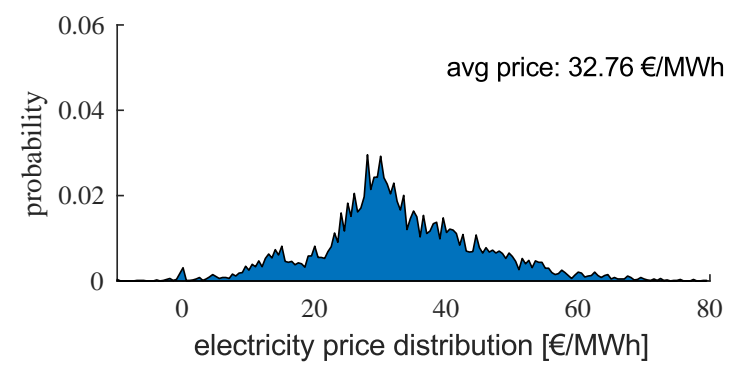

Fig. 4. Empirical day-ahead price distributions for Germany in 2014

For the parametrization of the models, an extensive set of fundamental data has been used, which have been assembled within the context of a dissertation [9]. These are fuel- and $\mathrm{CO} 2$ prices, must-run restrictions, technical and economic power plant parameters, nonavailability, feed-in time series of renewables and NTC capacities ${ }^{1}$ between market areas. It could be shown that the EULR-approach generates individual power plant schedules, exchange schedules and market prices especially when compared in a temporal resolution - that come very close to their empirical counterparts [9]. The resulting price distribution from the back-test optimization for the panEuropean power plant fleet 2014 is shown in figure 5. On average the price is underestimated by $0.51 € / \mathrm{MWh}$.

The analysis introduced above is extended in the following to show that the endogenous consideration of market coupling is crucial for market price quality. For this purpose the above mentioned fundamental data are used to parametrize the threestage approach. However, the first stage, which is solely used to generate the trading schedules between the market areas, is skipped. Instead, the exchange schedules generated in the calculation of the EULR-approach are used, to allow for a direct comparison of both approaches. As the German market area remains the focus of the analysis, the exchange flows

\footnotetext{
${ }^{1}$ Both models use the NTC approach since the implicit capacity allocation was switched to the flow-based approach in 2015 , it was necessary to choose the year 2014 for the back-test.
} 


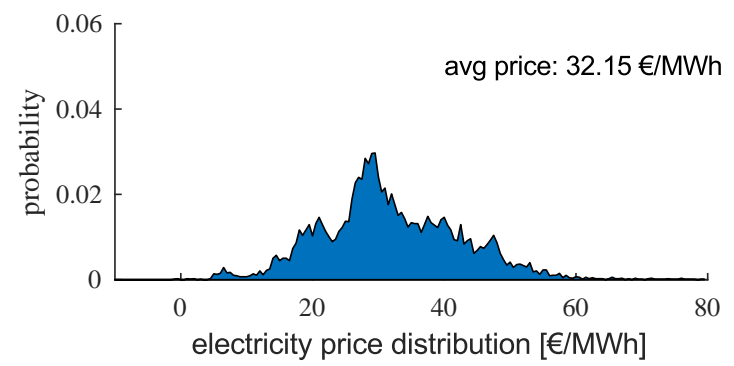

Fig. 5. Simulated price distributions for Germany in 2014 using EULR approach

between Germany and its neighboring countries are considered for the second stage by subtracting them from the residual load.

Although the power plant schedules do not differ significantly from the calculations of [9] or the real production quantities, the resulting prices are substantially different (see figure 6). Compared to EULR-prices in figure 5, the price

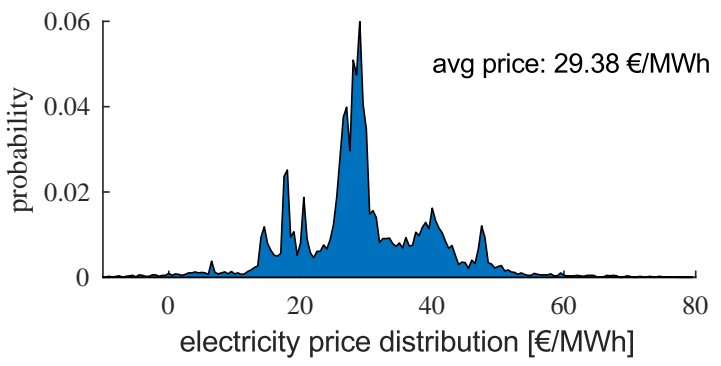

Fig. 6. Simulated price distributions for Germany in 2014 in a multi-stage approach using the exchange schedules calculated in the EULR-approach

distribution is less smooth in the three-stage approach. This behavior illustrates the facts discussed in section II, that there are inherent distortions of the merit order. Power plant bids from neighboring market zones are not considered in the subgradient method of the Lagrangian relaxation and, therefore, not in the price determination. As a result, there are de facto no power plants from neighboring markets that can be pricesetting ti the market area in focus. Thus, the resulting merit order is much less heterogeneous and the price distribution much more gradual than in the case of endogenous market coupling. As theoretically deduced in section II, this leads to a price decrease by $2.77 € / M W h$ on average.

When performing the EULR-calculation while fixing the exchange schedules (as in the multi-stage approach) the resulting price distribution (see figure 7) has a structurally similar characteristic to the one displayed previously in figure 6. The average price differs by $0.15 € / \mathrm{MWh}$, which results mainly due to differently parametrized gradient methods. This shows that the price impact is induced by endogenous marketcoupling.

The focus of the analysis in this paper is the resulting market price. Compared to the consideration of UC constraints according to [11] in the EULR approach, the three-stage

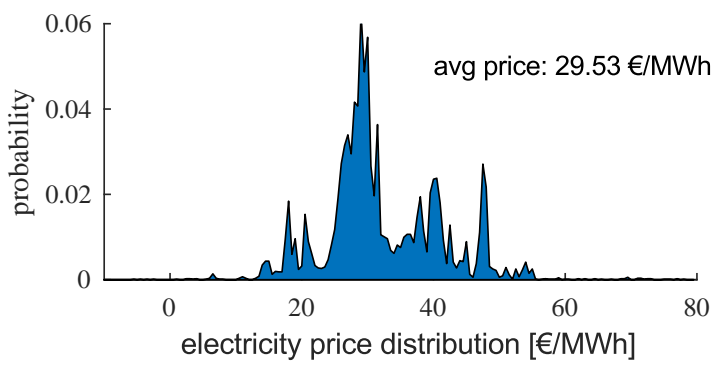

Fig. 7. Simulated price distributions for Germany in 2014 in the EULRapproach with fixed exchange schedules as used in figure 7

approach only considers simplified power plant constraints (see 1-3) whilst optimizing exchange flows. Therefore, the latter additionally distorts the exchange flows between the market areas [9].

\section{CONCLUSION}

We have shown that the separation between market coupling and unit commitment leads to the distortion of electricity prices, resulting in distorted incentives for dispatch in the individual market areas. This was simulated by conducting a backtest for the year 2014. Here, empirical electricity prices, cross-border volumes and other fundamental factors are available for use within the model. In contrast to a multistage approach the consideration of inter-zonal trading in a single-stage approach delivers more realistic electricity prices when applied to the pan-European power system. It was shown - both in theory and in simulation - that an endogenously considered market-coupling is the driving factor to the quality of resulting prices.

\section{REFERENCES}

[1] C. Mueller, "Verfahren zur langfristigen Planung der Energieversorgungsstruktur unter Berücksichtigung der Sektorenkopplung," Dissertation, RWTH Aachen University, 2020.

[2] T. Mirbach, "Marktsimulationsverfahren zur Untersuchung der Preisentwicklung im europäischen Strommarkt," Dissertation, RWTH Aachen University, 2009.

[3] S. Rahts, "Multi Level European Electricity Market Simulation using Network Flow Algorithm and Lagrangian Relaxation," Operations Research Proceedings, 2013.

[4] T. Drees, "Simulation des europäischen Binnenmarktes für Strom und Regelleistung bei hohem Anteil erneuerbarer Energien," Dissertation, RWTH Aachen University, 2016.

[5] C. Rehtanz, C. Spieker, D. Klein, B. Matthes, M.-L. Kloubert, "Begleitung Netzentwicklungsplan 2025 (NEMO VI)," 2018.

[6] D. Nailis, A. Nolde, M. Ritzau, "NEMO IV - Gutachten zumNetzentwicklungsplan 2024 im Auftrag der Bundesnetzagentur," 2015.

[7] Pöyry, "NEP2015 - Overview of market model BID3," 2015.

[8] F. Grote, A. Maaz, T. Drees, A. Moser, "Modeling of Electricity Pricing in European Market Simulations,", 12th International Conference on the European Energy Market (EEM), 2015.

[9] S. Raths, "Marktsimulationsverfahren für einen dezentral geprägten Strommarkt," Dissertation, RWTH Aachen University, 2019.

[10] NEMO Commitee, "Euphemia Public Description - Single Price Coupling Algorithm," 2019.

[11] M. Carrion, et al., A computationally efficient mixed-integer linear formulation for the thermal unit commitment problem, IEEE Transactions on Power Systems, vol. 21, no. 3, 1371-1378, 2006 